BULL. AUSTRAL. MATH. SOC.

VOL. $25(1982), 337-356$.

\title{
CLIQUE COVERINGS OF GRAPHS V: MAXIMAL-CLIQUE PARTITIONS
}

\author{
N.J, Pullman, H. Shank and W.D. Wallis
}

\begin{abstract}
A maximal-clique partition of a graph $G$ is a way of covering $G$ with maximal complete subgraphs, such that every edge belongs to exactly one of the subgraphs. If $G$ has a maximal-clique partition, the maximal-clique partition number of $G$ is the smallest cardinality of such partitions. In this paper the existence of maximal-clique partitions is discussed - for example, we explicitly describe all graphs with maximal degree at most four which have maximal-clique partitions - and discuss the maximal-clique partition number and its relationship to other clique covering and partition numbers. The number of different maximal-clique partitions of a given graph is also discussed. Several open problems are presented.
\end{abstract}

\section{Introduction and summary}

For our purposes, graphs have no self-adjacent vertices and no multiple edges. Complete subgraphs of a graph are called cliques. The number of vertices in a clique is its order. A clique of order $n$ is also called an n-clique or a $K_{n}$. A clique covering of $G$ is a family $C$ of cliques of $G$ such that every edge of $G$ lies in some member of $C$. If the members of $C$ are pairwise edge-disjoint, then $C$ is called a clique partition. If the clique covering $C$ has cardinality $|C|$ and

Received 9 Irovember 1981. We would like to thank Professor David A. Gregory for many helpful conversations. This work was supported in part by the llatural Sciences and Engineering Research Council of Canada under grant A4041 and by the Queen's University Advisory Research Council. 
$\left|C^{\prime}\right| \geq|C|$ for all clique coverings $C^{\prime}$ of $G$, then $C$ is called a minimum clique covering and $|C|$ is called the clique covering number of $G$, denoted by $\operatorname{cc}(G)$. Similarly $c p(G)$, the clique partition number of $G$, is the cardinality of a minimum clique partition. If $G$ has no edges, we define $\operatorname{cc}(G)=\mathrm{cp}(G)=0$. Since every clique partition is also a clique covering we have $\operatorname{cc}(G) \leq \mathrm{cp}(G)$ for all graphs $G$. Equality holds when (but not only when) $G$ is triangle-free, in which case $\operatorname{cc}(G)=\operatorname{cp}(G)=\varepsilon(G)$ the number of edges of $G$.

This subject has its origins in the problem of representing set intersections by graphs - see Erdös, Goodman and Pósa [2], Lovász [8], Harary [6] - and its matrix-theoretic form was studied by Ryser [15], [16]. Recently Orlin [10], de Caen [12], [13], Donald [1], [14] and Pullman [11], [12], [13], [14] have developed the subject further.

When seeking a minimum clique covering we may restrict our attention to maximal cliques (those contained in no other clique in $G$ ). The same is not true for clique partitions. In fact many graphs have no maximalclique partition (no partition of the edge-set into maximal cliques). For example if $n \geq 4$, then the graph obtained by deleting one edge from $K_{n}$ has none.

The purpose of this paper is to present some facts and open questions that we have found about maximal-clique partitions.

We discuss their existence in Section 2. For example, we describe explicitly all graphs of maximum degree $\Delta(G) \leq 4$ that have maximal-clique partitions (Theorem 1 ). We also present an alternative characterization (Theorem 2) which yields some sufficient conditions for the existence of such partitions.

If a graph $G$ has a maximal-clique partition, it would be natural to ask for the minimum cardinality of such partitions, the maximal-clique partition number of $G$, denoted by $\operatorname{mcp}(G)$. (For convenience we define $\operatorname{mcp}(G)=0$ if $G$ has no edges.) Clearly whenever $\operatorname{mcp}(G)$ is defined we have $\operatorname{cc}(G) \leq \operatorname{cp}(G) \leq \mathrm{mcp}(G)$ with equality when, for example, $G$ is triangle-free. We would expect to find that $\operatorname{cp}(G)<\operatorname{mcp}(G)$ for some (if not most) graphs, just as we would expect $c c(G)<\operatorname{cp}(G)$ usually. It is a curious fact that actually $\operatorname{cc}(G)=\operatorname{cp}(G)=\operatorname{mcp}(G)$ whenever $\Delta(G) \leq 4$ and $\operatorname{mcp}(G)$ is defined. This is discussed in Section 2 also. 
(An example where $\operatorname{cc}(G)<\operatorname{cp}(G)<\operatorname{mcp}(G)$ is given in Section 4.)

In Section 3, we discuss the number $M(G)$ of maximal-clique partitions of $G$. Forr example, we show, in Theorem 3, that for every $k \geq 0$, there exists a graph $G$ with $M(G)=k$. We also study the maximum value of $M(G)$ over all graphs on $n$ vertices.

In Section 4, we present a large class of graphs each having exactly two maximal-clique partitions. We use them to construct various examples and counter-examples concerning $\operatorname{mcp}(G)$.

\section{On the existence of maximal-clique partitions}

If $H$ is a subgraph which does not contain all of the edges of $G$, then we say that $H$ is a proper subgraph. If a subgraph $H$ has the property that for every clique $K$ in $G$, either every edge of $K$ or no edge of $K$ lies in $H$, then we say that $H$ separates the cliques of $G$. If some proper non-empty subgraph separates the cliques of $G$, we say $G$ is clique-separable. Otherwise $G$ is clique-inseparable. If a subgraph $B$ separates the cliques of $G$, but no proper non-empty subgraph of $B$ does so, we call $B$ a clique-block of $G$. Note that $B$ is cliqueinseparable in itself. Therefore a subgraph $B$ is a clique-block of $G$ if and only if $B$ is a clique-inseparable graph and $B$ is not a subgraph of any other clique-inseparable subgraph of $G$. Note that if $G$ has isolated vertices, then they form one clique-block of $G$. It was shown in [11, Lemma 2.7] that the family $B(G)$ of clique-blocks partitions the edge set of $G$. Therefore the family $M(G)$ of maximal cliques of $G$ is also partitioned by $B(G)$ and hence:

LEMMA 2.1. $G$ has a maximal-clique partition if and only if each of its clique-blocks has a maximal-clique partition, in which case

$$
\operatorname{mcp}(G)=\sum_{B \in B} \operatorname{mcp}(B)
$$

Therefore the study of maximal-clique partitions could be confined to clique-inseparable graphs.

The next lemma (Theorem 1 of [11]) gives an explicit description of all the clique-inseparable graphs $G$ with maximal degree $\Delta(G) \leq 4$. We refer the reader to [11] for the proof. 
LEMMA 2.2. If $G$ is clique-inseparable and $\Delta(G) \leq 4$, then $G$ is $\bar{K}_{n}$ (the graph on $n$ vertices with no edges), $K_{2}, K_{5}$, or one of the graphs

$$
\begin{array}{r}
G_{1}, G_{2}, \ldots, G_{10} ; P_{1}, P_{2}, \ldots, P_{j}, \ldots ; P_{6}^{\prime}, P_{7}^{\prime}, \ldots, P_{j}^{\prime}, \ldots ; \\
C_{4} ; C_{7}, C_{8}, \ldots, c_{j}, \ldots
\end{array}
$$

described in Figure 1.
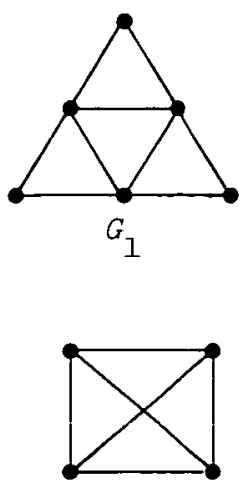

$G_{6}$

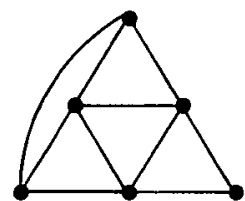

$G_{2}$
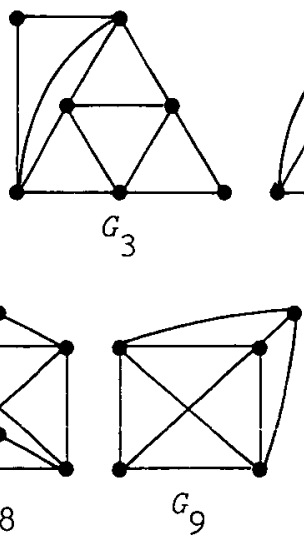
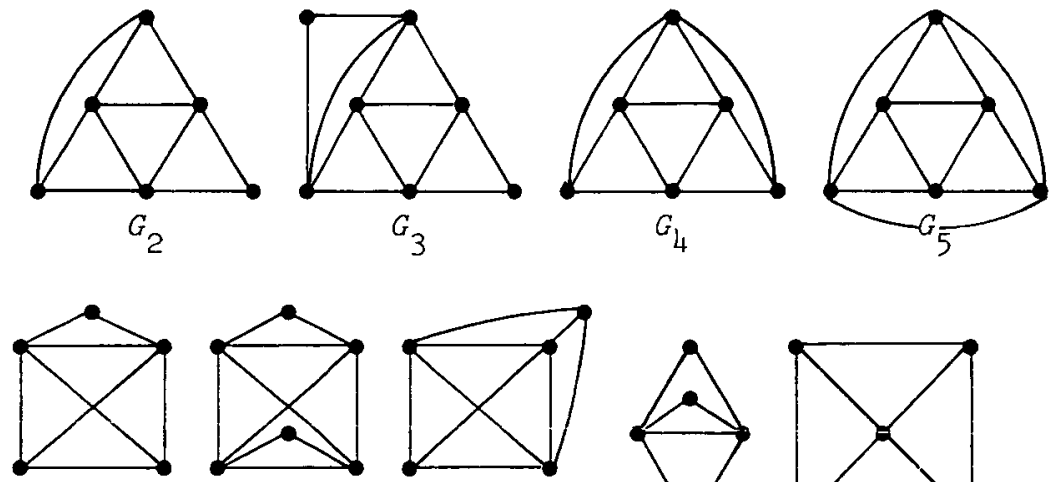

$G_{7}$

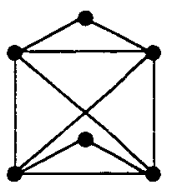

$G_{8}$

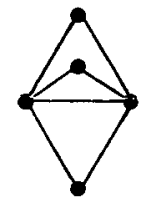

$G_{10}$

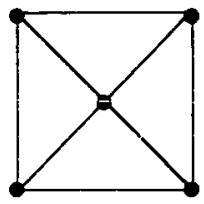

$c_{4}$
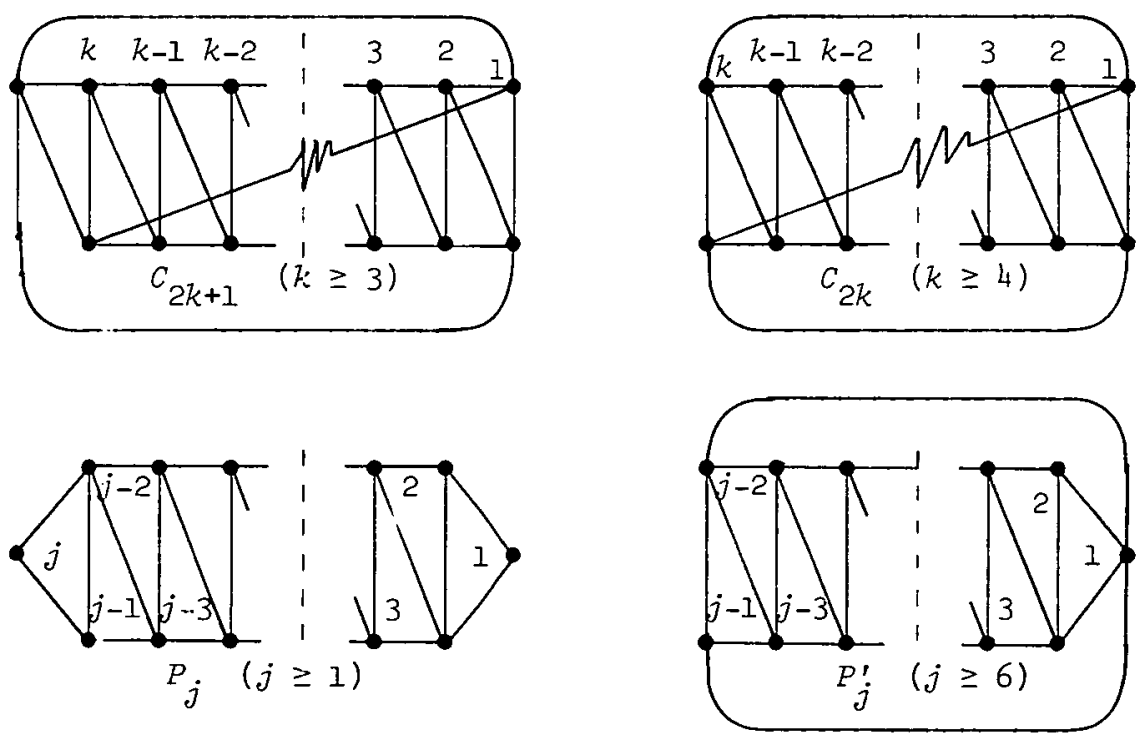

FIGURE 1 
The following is an immediate consequence of Lemmas 2.1 and 2.2.

THEOREM 1. If $G$ has no isolated vertices and $\Delta(G) \leq 4$, then $G$ has a maximal-clique partition if and only if its clique-blocks are either $G_{1}, G_{3}, G_{5}$ or cliques.

The graph of Figure 2, below, has eight clique-blocks: four $K_{2}$ 's , one $K_{3}$, one $G_{1}$ and two $G_{3}$ 's. Therefore by Theorem 1 , it has a maximal-clique partition. Although it would appear that Theorem 1 might be difficult to apply to more complicated graphs, there are linear-time algorithms for finding the clique-blocks of graphs $G$ with $\Delta(G) \leq 4$; see $[11]$.

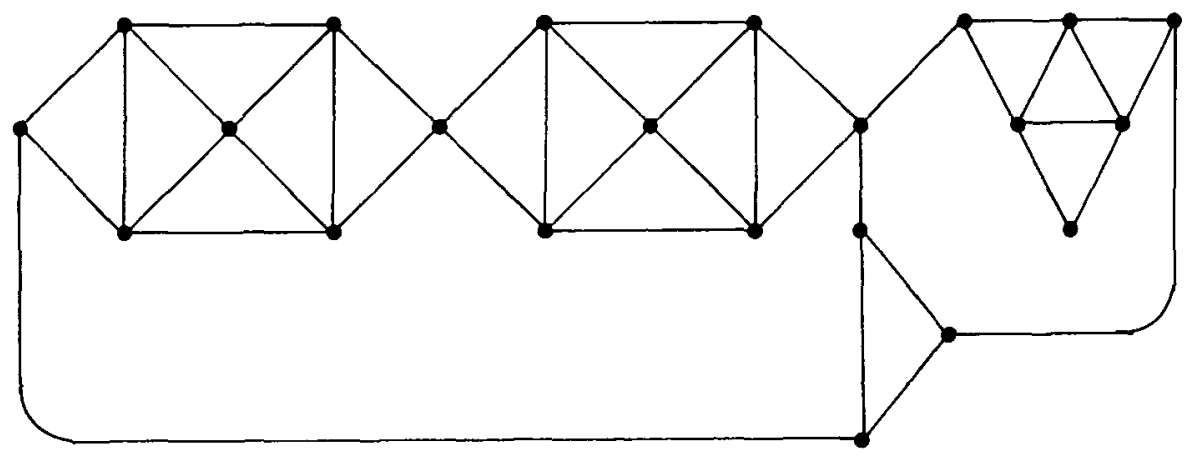

FIGURE 2

It is a consequence of Lemma 2.2 that all clique-inseparable graphs $G$ with $\Delta(G) \leq 4$ have at most one maximal-clique partition except for $G=G_{5}$ which has exactly two, and when such a $G$ has a maximal-clique partition it is also a minimum clique covering. Summarizing, we have:

COROLLARY 1.1. Whenever $\Delta(G) \leq 4$ and $G$ has a maximal-clique partition:

(a) $\operatorname{cc}(G)=\operatorname{cp}(G)=\operatorname{mcp}(G)$;

(b) all maximal-clique partitions of $G$ have the same cardinality; and

(c) $G$ has exactly $2^{2}$ maximal-clique partitions for some integer $l \geq 0$.

In Sections 3 and 4 we will show that $(a),(b)$ and $(c)$ are not 
typical, in that:

(1) there exist graphs $G$ with $\operatorname{cc}(G)<\operatorname{cp}(G)<\operatorname{mcp}(G)$ (see Example 4.3);

(2) there exist graphs having maximal-clique partitions of different cardinality (see Example 4.2); and

(3) for every $k>0$, there exist graphs having exactly $k$ maximal-clique partitions (see Theorem 3 ).

Although we were unable to obtain an explicit description of all graphs having maximal-clique partitions (except when the maximum degree is less than 5) we can present an alternative characterization. This provides a different perspective on the problem of existence of maximalclique partitions and provides some sufficient conditions.

First, we associate a graph $G^{*}$ with $G$ as follows. The vertices of $G^{*}$ are the maximal cliques of $G$. Two distinct vertices of $G^{*}$ are deemed adjacent in $G^{*}$ if they share an edge as cliques of $G$. We may assume without loss of generality that every edge in $G$ lies in at least two maximal cliques. The set of all maximal cliques of $G$ containing the edge $e$ of $G$ are the vertices of a clique in $G^{*}$. Call that clique $K^{*}(e)$.

THEOREM 2. A fomily A of maximal cliques of $G$ is a maximalclique partition of $G$ if and only if $A$ is an independent set of vertices in $G^{*}$ with the property that for every edge $e$ of $G$, some vertex of $K^{*}(e)$ is in A.

COROLLARY 2.1. If every edge of $G$ belongs to exactly two maximal cliques, then the following statements are equivalent:

(a) $G$ has a maximal-clique partition;

(b) $G$ has two maximal-clique partitions, which partition the maximal cliques of $G ;$ and

(c) $G^{*}$ is bipartite.

Proof. Use Theorem 2 to prove (c) implies (b) and to prove (a) implies (c).

NOTE. There may be more than one pair of maximal-clique partitions satisfying Corollary $2.1(b)$, unless $G^{*}$ is connected. Indeed if $G^{*}$ has 
$w$ connected components, then there are $2^{w-1}$ such pairs.

COROLLARY 2.2. Let $G$ be a triangulation of the plane containing no $K_{4}$ in which each edge belongs to exactly two triangles. Then $G$ has a maximal-clique partition (and hence two of them) if and only if $G$ is Eulerian.

Proof. Note that $G^{*}$ is a planar dual of $G$, so that $G^{*}$ is bipartite if and only if $G$ is Eulerian.

To what extent is the hypothesis of Corollary 2.1 necessary?

EXAMPLE 2.1. Let $B_{0}$ be the graph illustrated in Figure 3. For each $k>0$, add $2 k$ vertices to the circle in $B_{0}$ and add $4 k$ edges, linking the $2 k$ vertices to 0 and $0^{\prime}$. According to Corollary 2.2, the resulting graph $B_{k}$ has no maximal-clique partition. Nevertheless, every edge in the graph lies in two maximal cliques (triangles).

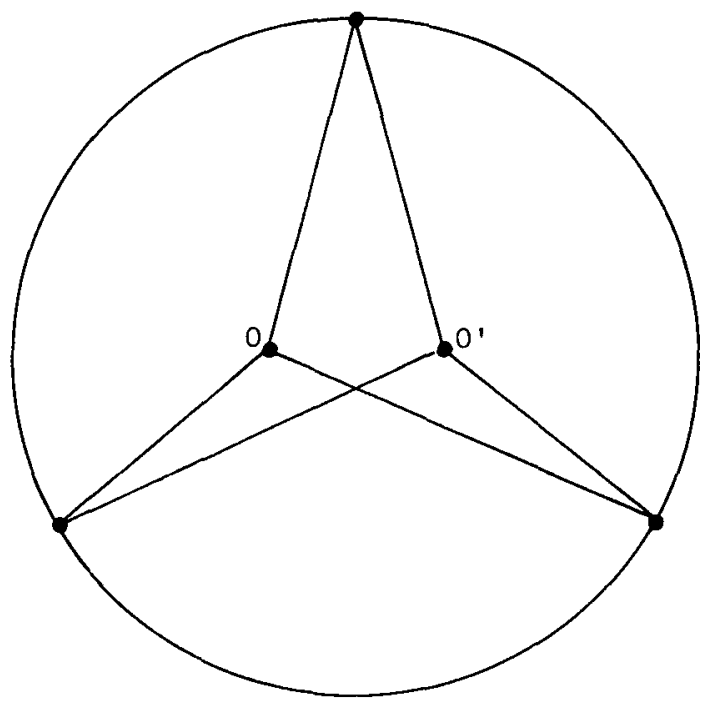

FIGURE 3 ,

We say that $G$ is $r$-partitionable if the family of all maximal cliques of $G$ can be partitioned into $r$ maximal-clique partitions.

The following is a partial generalization of Corollary 2.1.

COROLLARY 2.3. If for some $r \geq 1$, every edge of $G$ belongs to 
exactly $r$ maximal cliques, then $G$ is r-partitionable if and only if $G^{*}$ is r-colourable, in which case $G^{*}$ is r-chromatic.

Proof. If $G^{*}$ is r-colourable let $A_{1}, A_{2}, \ldots, A_{r}$ be its colour classes. If $e$ is any edge of $G$ and $l \leq i \leq r$, then one vertex of the $r$-clique $K^{*}(e)$ must be in $A_{i}$. Therefore each $A_{i}$ is a maximal-clique partition by Theorem 2. Since all the vertices of $G$ are coloured, $G$ is $r$-partitionable. Conversely, suppose $G$ is $r$-partitionable and $A_{1}, A_{2}, \ldots, A_{r}$ are its maximal-clique partitions. These define $r$ colour classes in $G^{*}$ hence $\chi\left(G^{*}\right) \leq r$. But $K^{*}(e)$ is an $r$-clique, hence $X\left(G^{*}\right)=r$.

Corollary 2.3 shows that $G$ is $r$-partitionable for at most one value of $r$. Notice that $r$-partitionable graphs have the property that every edge lies in exactly $r$ maximal cliques. In the next section we shall encounter graphs $H_{k}$ which have some edges in 2 maximal cliques and others in $k$ maximal cliques. Thexefore these graphs cannot be $r$-partitionable (when $k>2$ ) even though they have maximal-clique partitions.

EXAMPLE 2.2. Let $L_{m}$ be the complete tripartite graph on $3 m$ vertices defined by 3 edge-free m-sets for $m \geq 1$; then $L_{m}$ has precisely $m$ maximal-clique partitions (and these partition the maximal cliques of $\left.L_{m}\right)$. To see why this is so, we apply Corollary 2.3. The maximal cliques are all $\mathrm{m}^{3}$ of the triangles in $L_{m}$. Each edge lies on exactly $m$ maximal cliques (triangles). Now let $U=\left\{U_{i}: 1 \leq i \leq m\right\}$, $V=\left\{V_{i}: 1 \leq i \leq m\right\}$ and $W=\left\{W_{i}: 1 \leq i \leq m\right\}$ denote the three independent disjoint vertex sets defining $L_{m}$ and let $T(i, j, t)$ be the triangle whose vertices are $U_{i} V_{j} W_{i+j+t}$ for $1 \leq i, j, t \leq m$. The subscript on $W$ is read modulo $m$. Each set $A_{t}=\{T(i, j, t): 1 \leq i, j \leq m\}$ is independent in $L_{m}^{*}$. To verify this, suppose $T(i, j, t)$ has an edge in common with $T\left(i^{\prime}, j^{\prime}, t\right)$ so $(i, j)=\left(i^{\prime}, j^{\prime}\right),(i, i+j+t)=\left(i^{\prime}, i^{\prime}+j^{\prime}+t\right)$ or $(j, i+j+t)=\left(j^{\prime}, i^{\prime}+j^{\prime}+t\right)$; hence $i=i^{\prime}, j=j^{\prime}$ and 
$T(i, j, t)=T\left(i^{\prime}, j^{\prime}, t\right)$. It also is easy to check that every vertex of $L_{m}^{*}$ is some $T(i, j, t)$. Therefore $\left\{A_{1}, A_{2}, \ldots, A_{m}\right\}$ is an m-colouring of $L_{m}^{*}$, and hence $L_{m}$ is m-partitionable by Corollary 2.3 .

Example 2.2 establishes

COROLLARY 2.4. There exists an r-partitionable graph for every $r \geq 1$.

\section{Graphs with a prescribed number of maximal-clique partitions}

The graph $G_{5}$ of Figure 1 has two maximal-clique partitions, that is $M\left(G_{5}\right)=2$. Specify one triangle $t$ in $G_{5}$. Let $C$ be the clique partition containing $t$ and $\mathrm{D}$ be the other. Let $H_{k}$ be the graph obtained by joining $k$ replicas $G_{5}^{l}, G_{5}^{2}, \ldots, G_{5}^{k}$ of $G_{5}$ together at $t$. Figure 4 (see p. 346) illustrates $H_{3}$. Let $C_{i}$ and $D_{i}$ be the maximalclique partitions in $G_{5}^{i}$ corresponding to $C$ and $D$ respectively in $G_{5}$. Define the maximal-clique partitions $E_{0}=\underset{i=1}{U} C_{i}$ and $E_{j}=D_{j} \cup\left(E_{0} \backslash C_{j}\right)$ for $j=1,2, \ldots, k$. Suppose $E$ is any maximalclique partition of $G$. If $t \in E$, then $E=E_{0}$. If $t \notin E$, let $e$ be an edge of $t$. The edge $e$ must be covered by some triangle of $G-t$ which must be in some $D_{j}$, and hence all of $D_{j}$ is in $E$. The presence of the triangles of $D_{j}$ in $E$ precludes the presence of any triangles of $D_{i}$ in $E$ for $i \neq j$. Therefore the edges of $H$ not covered by $D_{j}$ must be covered by the triangles of $C_{i} \backslash t$ with $i \neq j$. Consequently $E=E_{j}$. This shows that $M\left(H_{k}\right)=k+1$ for all $k \geq 2$. As we have already seen some graphs with 0 and 1 maximal-clique partitions, we have shown:

THEOREM 3. For every $k \geq 0$, there exists a graph with exactly $k$ maximal-clique partitions.

Let $G(n, k)$ denote the set of all graphs $G$ on $n$ vertices with 


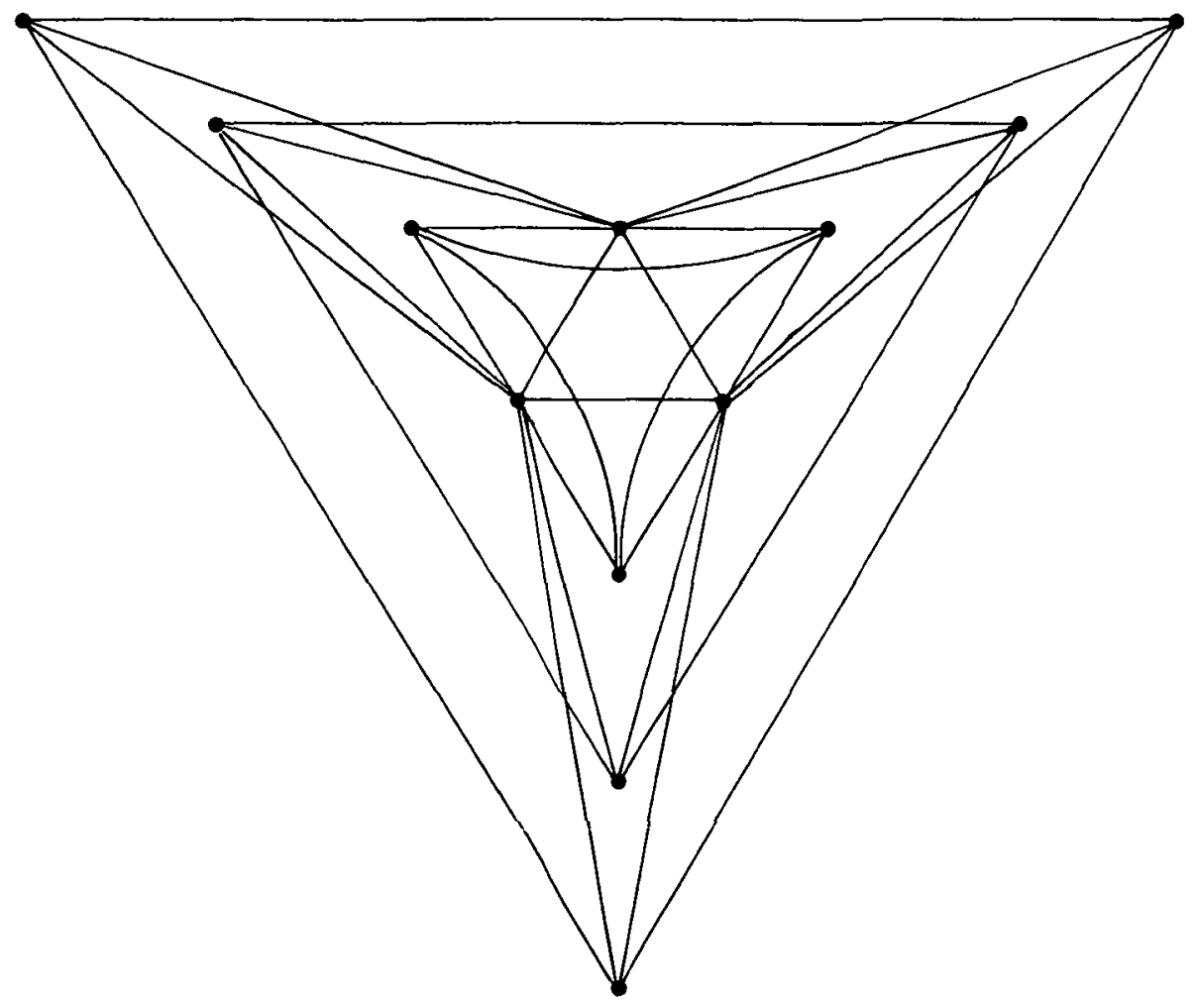

FIGURE 4

exactly $k$ maximal-clique partitions. That is, $G$ is in $G(n, k)$ if and only if $M(G)=k$ and $v(G)=n$. According to Theorem 3, given any $k \geq 0$, there exist $n$ such that $G(n, k)$ is not empty.

Let $M(n)$ denote the largest $k$ for which $G(n, k)$ is not empty. our previous results imply that $M(n)=1$ for all $n \leq 5$ and $M(6)=2$. The graph with $m$ connected components, each isomorphic to $G_{5}$ (see Figure 1 ), has $6 m$ vertices and precisely $2^{m}$ maximal-clique partitions, from which it follows that $M(n) \geq 2^{\lfloor n / 6\rfloor}$. But we can improve on that bound.

We write $k(k, m)$ for the complete $k$-partite graph with each part of size $m$ : the vertices of $K(k, m)$ are partitioned into $k$-sets $V_{1}, V_{2}, \ldots, V_{k}$, and vertices are adjacent if and only if they lie in 
different m-sets. The graph $L_{m}$ of Example 2.2 is $K(3, m)$. Let $M(K(k, m))=M(k, m)$ be the number of maximal-clique partitions of $K(k, m)$.

A maximal clique in $K(k, m)$ is a $k$-clique with one vertex in each part. Therefore a maximal-clique partition may be represented as a $k \times m^{2}$ array $A$ where each column represents a maximal clique: $A(i, j)=l$ means that clique $j$ contains vertex $l$ from $V_{i} ;$ and given two rows $i_{1}$ and $i_{2}$, the array formed by deleting all other rows of $A$ contains every possible column of two entries from $\{1,2, \ldots, m\}$ exactly once. Such an object is an orthogonal array $\mathrm{OA}\left[m^{2}, k, m, 2\right]$ of index 1 , strength 2 with $m$ constraints and $k$ levels. So we seek the number of different $\mathrm{OA}\left[\mathrm{m}^{2}, k, m, 2\right]$ 's (an orthogonal array derived from another by column permutations will not be considered different).

An orthogonal array of strength 2 with $k$ levels is equivalent to an ordered set of $k-2$ orthogonal Latin squares of side $m$; rows 1 and 2 of the array are used to coordinatize the squares as two sets of Latin squares are different if and only if the two corresponding arrays cannot be obtained from each other by column permutations. Therefore $M(k, m)$ is the number of different ordered sets of $k-2$ mutually orthogonal Latin squares of order $m$.

A reduced Latin square on the symbols say $\{1,2, \ldots, m\}$ is one with first row and column in standard order say $(1,2, \ldots, m)$. Let $z_{m}$ denote the number of reduced Latin squares on $m$ symbols; then, from [5],

$$
z_{m} \geq(m-2) !(m-3) ! \ldots 1 !
$$

so

$$
\begin{aligned}
M(3, m) & =m !(m-1) ! \tau_{m} \\
& \geq m !(m-1) ! \ldots I ! \text { for all } m .
\end{aligned}
$$

So, given $n$ divisible by 3 , there is an $n$-vertex graph $G_{n}$ with at least $(n / 3) !((n / 3)-1) ! \tau_{n / 3}$ maximal-clique partitions. By attaching one end of one (or two) $K_{2}$ 's to different vertices of $G_{n}$ we obtain graphs with the same number of maximal-clique partitions, having $n+1$ (or 
$n+2$ ) vertices. This establishes

THEOREM 4. $M(n) \geq m !(m-1) ! z_{m}$ where $m=\lfloor n / 3\rfloor$.

The following table gives some idea of how fast $M(n)$ grows: (see [3] for $z_{i}$ with $i \leq 5$, [4] for $z_{6}$, [17] for $z_{7}$ and [19] for $z_{8}$ ).

TABLE 1

\begin{tabular}{|c|c|c|c|c|c|c|c|c|}
\hline$m$ & 1 & 2 & 3 & 4 & 5 & 6 & 7 & 8 \\
$z_{m}$ & 1 & 1 & 1 & 4 & 56 & 9,408 & $16,942,080$ & $535,281,401,856$ \\
$M(3 m)$ & 1 & 2 & $\geq 12$ & $\geq 576$ & $\geq 161280$ & $\geq 812,851,200$ & $\geq 6 \times 10^{13}$ & $\geq 1 \times 10^{20}$ \\
\hline
\end{tabular}

Except for $m \leq 2$, Theorem 4 was used for the lower bound on $M(3 m)$. The lower bound on $M(9)$ is also obtained from the graph $L_{3}$ of Example 2.2.

\section{Proper intersection graphs}

Let $S$ be a set of $n \geq 1$ elements and $S_{r}$ be the family of all $r$-sets in $S$ for $1 \leq r \leq n$. Let $V$ be any nonempty subset of $S_{r}$ and declare two members of $V$ to be adjacent if their intersection has cardinality $r-1$. We call the resulting graph $G(S, V)$ the intersection graph generated by $S$ and $V$. For example, all line graphs are intersection graphs. The triangle graphs (see [11]) are also intersection graphs.

Fix $S$ and $V$ arbitrarily and let $G=G(S, V)$. If $W$ is any $(r-1)$-set in $S$, let $A(W)=\{T \in V: W \subset T\}$ and if $A(W) \neq \emptyset$, let $A(W)$ be the subgraph of $G$ induced by $A(W)$; that is, $A(W)=G[A(W)]$. Define $A=\left\{A(W): W \in S_{r-1}\right\}$. If $Z$ is any $(r+1)$-set in $S$, let $\mathbf{B}(Z)=\{T \in V: T \subset Z\}$ and if $B(Z) \neq \emptyset$, let $B(Z)=G[B(Z)]$. Define $\mathbf{B}=\left\{B(Z): Z \in S_{r+1}\right\}$. The definition of adjacency in $G$ implies that $A$ is a clique covering. Suppose $W \neq W^{\prime}$ and $T_{1}, T_{2}$ are common vertices of $A(W)$ and $A\left(W^{\prime}\right)$; then for $i=1,2, T_{i}=W \cup\left\{x_{i}\right\}$ for some $x_{i} \notin W$ and $T_{i}=W^{\prime} \cup\left\{x_{i}^{\prime}\right\}$ for some $x_{i}^{\prime} \neq W^{\prime}$. We then have $T_{i}=\left(W \cap W^{\prime}\right) \cup\left\{x_{i}, x_{i}^{\prime}\right\}$ and hence $W W^{\prime}=\left\{x_{i}^{\prime}\right\}$. Therefore $T_{1}=T_{2}$. 
Consequently $A(W)$ and $A\left(W^{\prime}\right)$ have at most one common vertex. This establishes

LEMMA 4.1. If $G(S, V)$ is any intersection graph, then $A$ is a clique partition.

For each subset $Y$ of $S$, let $\bar{Y}$ denote $S \backslash Y$ and let

$\vec{V}=\{\bar{T}: T \in V\}$. For each $Z \in S_{p+1}$, let $\bar{A}(\bar{Z})=\{\bar{T} \in \bar{V}: \bar{Z} \subset \bar{T}\}$. The mapping $Y \rightarrow \bar{Y}$ preserves adjacency between $G(S, V)$ and $G(S, \bar{V})$, and maps $\bar{A}(\bar{Z})$ onto $B(Z)$. Therefore $B$ is a clique partition of $G$, as $\bar{A}(\bar{Z})$ is a clique partition of $G(S, \bar{V})$ by Lemma 4.1 .

LEMMA 4.2. Let $G(S, V)$ be any intersection graph. If $A$ is a member of $A$ and $B$ is a member of $B$, then $A \cap B$ has at most one edge.

Proof. Suppose $A=A(W), B=B(Z)$ and

$$
A(W) \cap B(Z)=\left\{T_{1}, T_{2}, \ldots, T_{k}\right\} .
$$

For each $i \geq I$ there exist $x_{i} \in S \backslash W$ and $y_{i} \in Z$ such that $T_{i}=W \cup\left\{x_{i}\right\}$ and $T_{i}=Z \backslash\left\{y_{i}\right\}$, by the definitions of $A(W)$ and $B(Z)$. We have $y_{i} \notin W \cup \bar{Z}$ and $W \cup \bar{Z}=S \backslash\left\{x_{1}, y_{1}\right\}$. Therefore $k \leq 2$.

We will say that an intersection graph is proper if every $(r-1)$-set in $S$ contained in at least one member of $V$ is contained in at least three different members of $V$, and every $(r+1)$-set in $S$ that contains a member of $V$ contains at least three different members of $V$. The order of every clique in $A$ and every clique in $B$ is at least 3 if $G(S, V)$ is proper. Moreover every vertex will have exactly $r$ members of $A$ and $n-r$ members of $B$ incident with it.

LEMMA 4.3. If $G(S, V)$ is a proper intersection graph then $A$ and B are disjoint maximal-clique partitions.

Proof. It follows from the previous lemma that $A \cap B=\varnothing$. Let $A(W)$ be any member of $A$. Since $G$ is proper we know that $A(W)$ has at least three vertices $T_{1}, T_{2}, T_{3}$. Suppose $T$ is any vertex of $G$ adjacent to each of them. Let $\left\{x_{i}\right\}=T_{i} \backslash W$ for $i=1,2,3$, so that $T \cap T_{i}=(T \cap W) \cup\left(T \cap\left\{x_{i}\right\}\right)$. If all three $x_{i}$ were in $T$, then 
$\left\{x_{1}, x_{2}, x_{3}\right\} \subseteq T$ so $|T \cap W| \leq r-3$ and hence $\left|T \cap T_{i}\right| \leq r-2$ which is impossible. Therefore some $x_{i} k T$, so that $|T \cap W|=r-1$ and hence $W \subseteq T$ Consequently $T$ is a vertex of $A(W)$. But $T$ was arbitrary and hence $A(W)$ is a maximal clique. By the same argument, the cliques of $G(S, \bar{V})$ whose vertex-sets are $\bar{A}(\bar{Z})$ are maximal. Therefore. $B(Z)$ is maximal because the mapping $Y \rightarrow \bar{Y}$ of $G(S, V)$ onto $G(S, \bar{V})$ is adjacency-preserving .

LEMMA 4.4. If $K$ is any clique in a proper intersection graph $G$ and $K$ has more than 2 vertices, then $K$ is covered by some member of $A$, or $K$ is covered by some member of $B$; but $K$ cannot be covered by a member of $A$ and by a member of $B$.

Proof. First, notice that Lemma 4.2 implies the last assertion of Lemma 4.4. Next we assume that $K$ is a triangle with vertices $T_{1}, T_{2}$, $T_{3}$. We have $T_{i}=W \cup\left\{x_{i}\right\}$ for some $W \in S_{r-1}$ and some $x_{i} \neq W$ for $i=1,2$ because $T_{1}$ is adjacent to $T_{2}$. Let $Z=T_{1} \cap T_{2} \cap T_{3}$. If $Z=W$, then $K \subseteq A(W)$. If $Z \neq W$, then $T_{3}=\left\{x_{1}, x_{2}\right\} \cup W \backslash\{y\}$ for some $y \in W$. Let $Q=T_{1} \cup T_{2}$. Then $Q \in S_{p+1}$ and $T_{i} \subseteq Q$ for $i=1,2,3$. Thus $K \subseteq B(Q)$. Therefore $K$ is covered by a member of A or by a member of B. This establishes Lemma 4.4 if $K$ is a triangle. Now suppose that $K$ is an arbitrary clique of order exceeding 3 and $T, T_{1}, T_{2}, T_{3}$ are among its vertices. We will use the notation $P Q R$ to denote the triangle whose vertices are $P, Q, R$. We know that the triangle $T_{1} T_{2} T_{3}$ is covered by a clique $J$ that belongs to $A$ or to $B$, say to $A$. We also know that $T_{1}{ }^{T} 2^{T}$ is covered by a member $M$ of $A$ or of $B$. We will show that $M$ must be in $A$ also. Suppose to the contrary, that $M \in \mathrm{B}$. The edge $T_{2} T_{3}$ is covered by some member of $\mathrm{B}$; call it $M^{\prime}$. Lemma 4.2 implies that $M \cap J$ has at most one edge, hence $M \cap J=T_{1} T_{2}$. Therefore $M^{\prime} \neq M$. Similarly, the edge $T T_{2}$ is covered by some member $J^{\prime}$ of $A$ and $J^{\prime} \neq J$. Now consider the triangle $K^{\prime} \equiv T T_{2} T_{3}$. Two of its edges $T_{2} T_{3}, T T_{2}$ are in different members of $A$ so $K^{\prime}$ cannot be covered by any member of $A$. Similarly $K^{\prime}$ cannot be contained in any member of $B$. But that contradicts the fact that Lemma 
4.4 holds for triangles. Therefore $T T_{1} T_{2}$ is covered by some member $J^{\prime \prime}$ of $A$, but $J^{\prime \prime} \cap J$ contains an edge $T_{1} T_{2}$ and $A$ is a partition of the edges of $G$, so $J^{\prime \prime}=J$ and hence $T T_{1} T_{2} \subseteq J$. Similarly $T T_{i} T_{j} \subseteq J$ for all $1 \leq i, j \leq 3$. Since $T$ was an arbitrary vertex of $K$ and $J$ was uniquely determined by $T_{1}, T_{2}, T_{3}$ it follows that $K \subseteq J$. This completes the proof of Lemma 4.4 .

Lemmas 4.3 and 4.4 are summarized in the following

THEOREM 5. Every proper intersection graph is two-partitionable.

EXAMPLE 4.1. If $|S|=n$ and $V=S_{p}$, let $G(n, r)$ denote $G(S, V)$. It is easy to verify that for all $1 \leq r \leq n, G(n, r)$ is an $r(n-r)$-regular graph on $\left(\begin{array}{l}n \\ r\end{array}\right)$ vertices and $\left(\begin{array}{l}n \\ 2\end{array}\right)\left(\begin{array}{l}n-2 \\ r-1\end{array}\right)$ edges, A consists of $\left(\begin{array}{c}n \\ r-1\end{array}\right)$ cliques of order $n-r+1$ and $B$ consists of $\left(\begin{array}{c}n \\ r+1\end{array}\right)$ cliques of order $r+1$. For $G(n, r)$ to be proper, we require $n-2 \geq r \geq 2$.

EXAMPLE 4.2. $G(5,2)$ is the line graph of $K_{5}$ (the complement of the Petersen graph). It has 10 vertices, $A$ consists of five 4-cliques and $B$ consists of 10 triangles. Therefore graphs can have maximalclique partitions of different cardinality. Is there a graph on fewer than 10 vertices having that property?

LEMMA 4.5. Suppose $A$ and $B$ are the maximal-clique partitions of a two-partitionable graph $G$. If $A \in A$, let $H$ be the graph obtained by replacing $A$ by a clique $A^{\prime}$ of higher order. Then $H$ has precisely one maximal-clique partition $A^{\prime}$, obtained by replacing $A$ by $A^{\prime}$ in A.

Proof. Evidently $A^{\prime}$ is maximal in $H, A^{\prime}$ is a maximal-clique partition of $H$ and $A^{\prime} \cup B$ partitions the maximal cliques of $H$. Let $M$ be any maximal-clique partition of $H$. The clique $A^{\prime}$ must be in $M$ because $\mathrm{M}$ covers $H$ and no member of $\mathrm{B}$ covers $A^{\prime}$. Now $M^{\prime}=\left(M \backslash A^{\prime}\right) \cup\{A\}$ is a maximal-clique partition of $G$ and hence is either $A$ or $B$. But $A \notin B$, hence $M^{\prime}=A$ and $M=A^{\prime}$.

EXAMPLE 4.3. The graph $G(5,3)$ has ten vertices and 30 edges. A consists of $10 K_{3}$ 's, B of $5 K_{4}^{\prime}$ 's. Replace one of the triangles in 
A by a new $K_{4}$ to obtain a graph $H$. By Lemma $4.5, \operatorname{mcp}(H)=10$. We shall now show that $\operatorname{mcp}(H)>\mathrm{cp}(H)$. The graph $H$ has 33 edges. If we let $C=B \cup Q$ where $Q$ is the set of 3 edges added to $G$ to form $H$, then $C$ is a clique partition of $H$ and $|C|=8$.

Next, we show that $\operatorname{cp}(H)=8$. Suppose that $\mathrm{D}$ is any clique partition of $H, c=|D|$ and $d_{j}$ is the number of $j$-cliques in $D$ for each $j \geq 2$. We have

(a) $d_{2}+3 d_{3}+6 d_{4}=33$ and

(b) $d_{2}+d_{3}+d_{4}=c$.

It follows from $(a)$ that $d_{2} \equiv 0(\bmod 3)$. Let $t=d_{2} / 3 ;$ then

(c) $d_{3}+2 d_{4}=11-t$ and

(d) $c=11+2 t-d_{4}$.

But $d_{3} \geq 0$, so (c) implies that $d_{4} \leq\lfloor(11-t) / 2\rfloor$. Therefore, applying (d), we see that $c \geq 8$ when $t \geq 1$. If $t=0$, then $d_{2}=0$, so $Q \cap D=\emptyset$. But the members of $Q$ must be covered by $D$, therefore $D$ contains $K$, the new 4-clique used to transform $G$ into $H$. There are only two $K_{4}$ 's in $G$ that do not share any of $K^{\prime}$ 's edges. Therefore $d_{3} \leq 2$. So $c \geq 9$ when $t=0$, by (d). Since $t \geq 0$, we have shown that $|D| \geq 8$ for any clique partition $D$ of $H$. But $|C|=8$. Therefore $\operatorname{cp}(H)=8$. Finally, we show that $\operatorname{cc}(H)=6$. Let $\mathrm{E}=\mathrm{B} \cup\{K\} ;$ then $\mathrm{E}$ is a clique covering of $H$ and $|\mathrm{E}|=6$. Any clique in $H$ is contained in some 4-clique, so any clique covering can be replaced by one of the same cardinality containing only 4-cliques. But all the 4-cliques of $H$ lie in $E$ and no proper subfamily of $E$ covers $H$. Thus $\operatorname{cc}(H)=6$. Therefore $\operatorname{cc}(H)<\operatorname{cp}(H)<\operatorname{mcp}(H)$. Is there a graph $G$ on fewer than 11 vertices having $\operatorname{cc}(G)<\operatorname{cp}(G)<\operatorname{mcp}(G)$ ?

EXAMPLE 4.4. Let $S$ be the set of points in $P G(m, s)$, the projective $m$-space of order $s$. Let $V_{r}$ be the family of all independent $r$-sets in $S$ for $1 \leq r \leq m+1$, that is, an r-set of $S$ is in $V_{r}$ if it is contained in no $(r-2)$-flat of the geometry. The 
intersection graph $P(m, s, r)=G\left(s, v_{p}\right)$ is proper if $r \geq 3$. For any $r \geq 1, P(m, s, r)$ has $\nu(m, s, r)$ vertices where

$$
v=s^{\left(\begin{array}{l}
r \\
2
\end{array}\right)}\left(s^{m+1}-1\right)\left(s^{m}-1\right) \ldots\left(s^{m-r+2}-1\right) / r !(s-1)^{r}
$$

This formuia for $\left|v_{r}\right|=v(m, s, r)$ is derived in [18, p. 52]; note that the factor $(k+1)$ ! was omitted from the denominators of the last expression on page 52 and the first expression on page 53. Given any $(r-1)$-set $W$ of $S, A(W)=\emptyset$ if $W$ is dependent. If $W$ is independent, let $F$ denote the $(r-2)$-flat generated by $W$; then $|A(W)|$, the number of independent r-sets containing $W$, is $|S \backslash F|=\left(s^{m+1}-1\right) /(s-1)-\left(s^{r-1}-1\right) /(s-1)$. Therefore every member of $A$ is a clique of order $t \equiv\left(s^{m+1}-s^{r-1}\right) /(s-1)$. But just before Lemma 4.3 we noted that every vertex of a proper intersection graph is incident with precisely $r$ cliques of $A$ when its vertices are $r$-sets. It follows that the degree of each vertex of $P(m, s, r)$ is $d=r(t-1)$ and hence for all $r \geq 1, P(m, s, r)$ is regular of degree $r\left(s^{m+1}-s^{r-1}-s+1\right) /(s-1)$. We also know that $|A|=v(m, s, r-1)$ because the members of $A$ are in a one-to-one correspondence with the independent $(r-1)$-sets. The $B$ cliques are not necessarily all of the same order. For example, B consists of 28 3-cliques and 74 -cliques in $P(2,2,3)$.

Similar examples can be obtained by using affine m-space of order $s$ for $S$.

EXAMPLE 4.5. Tree graphs of matroids (matroid basis graphs) are further examples of intersection graphs. The following is a paraphrase of the definition given by Holzmann and Harary [7]. Let $S$ be a finite, nonempty set and $V$ a family of subsets of $S$ satisfying

(1) no member of $V$ properly contains another, and

(2) if $T_{1}, T_{2} \in V$, then for every $x \in T_{1}$ there exists $y \in T_{2}$ such that $\left(\left(T_{1} \backslash\{x\}\right) \cup\{y\}\right) \in V$.

The members of $V$ are called the bases of $M \equiv(S, V)$. Next, let $T(M)$ be the graph whose vertices are the bases of $M$, with two bases $T, T^{\prime}$ 
deemed adjacent if for some $x, y$ in $S, T \backslash\{x\}=T^{\prime} \backslash\{y\}$. Axiom (2) ensures that all bases of $M$ have the same cardinality, $r$. The definition of adjacency implies that two bases are adjacent if and only if their intersection has cardinality $r-1$. Therefore $T(M)$ is the intersection graph $G(S, V)$.

To what extent is the converse true? Matroid basis graphs are connected (see, for example, [9]), but not all intersection graphs are connected. (It is easy to see that $G(S, V)$ is disconnected if and only if for some proper subset $V_{1}$ of $V,\left|T_{1} \cap T_{2}\right|<r-1$ for all $T_{1} \in V_{1}$ and all $\left.T_{2} \in V \backslash V_{1}.\right)$ However, even if we limit our attention to connected intersection graphs $G(S, V)$ we can find examples for which $(S, V)$ does not satisfy axiom (2). The following is such an example.

EXAMPLE 4.6 . Let $S=\{1,2,3, \ldots, 18\}$ and $A_{i}=\{x \in S: x \equiv i(\bmod 3)\}$ for $i=0,1$ and 2 . Let $V$ be the family of all 6-sets $T$ in $S$, except those such that $\left|T \cap A_{0}\right|=\left|T \cap A_{1}\right|=2$. Evidently $G(S, V)$ is a proper intersection graph. It can be shown that this graph is connected. (First show that the subgraph $H$ generated by $\bigcup_{i=0}^{2}\left\{T \in V: A_{i} \cap T=\not\right\}$ is connected, then show that every member of $\bigcup_{i=0}^{2}\left\{T \in V:\left|T \cap A_{i}\right|=2\right\}$ is connected to $H$.$\} On the other hand, if we let T_{1}=\{3,1,4,2,5,8\}$ and $T_{2}=A_{0}$, then $T_{1}, T_{2}$ are in $V$ but

$$
\left(\left(T_{1} \backslash\{8\}\right) \cup\{y\}\right) \neq V \text { for all } y \in T_{2} \text {. }
$$

Therefore $(S, V)$ does not satisfy axiom (2).

\section{References}

[1] Alan Donald, "Edge and arc partitions of arbitrary graphs and digraphs" (MSc Thesis, Queen's University, Kingston, Canada, 1979). 
[2] Paul Erdös, A.W. Goodman, and Louis Pósa, "The representation of a graph by set intersections:", Canad. J. Math. 18 (1966), 106-112.

[3] Leonhard Euler, "Recherches sur une nouvelle espèce de quarrés magiques", Verh. Zeerwsch. Genootsch. Wetensch. Vlissingen 9 (1782), 85-239.

[4] R.A. Fisher and F. Yates, "The $6 \times 6$ Latin squares", Proc. Cambridge Philos. Soc. 30 (1933-1934), 492-507.

[5] Marshall Hall, Jr., "Distinct representatives of subsets", BuZZ. Amer. Math. Soc. 54 (1948), 922-926.

[6] Frank Harary, Graph theory (Addison-Wesley, Reading, Massachusetts; Menlo Park, California; London; 1969).

[7] Carlos A. Holzmann and Frank Harary, "On a tree graph of a matroid", SIAM J. Appl. Math. 22 (1972), 187-193.

[8] L. Lovász, "On covering of graphs", Theory of graphs, 231-236 (Proc. Colloq. Tihany, 1966. Academic Press, New York and London, 1968).

[9] Stephen B. Maurer, "Matroid basis graphs. I", J. Combin. Theory Ser. B 14 (1973), 216-240.

[10] James Orlin, "Contentment in graph theory: covering graphs with cliques", Neder. Akad. Wetensch. Proc. Ser. A 80 = Indag. Math. 39 (1977), 406-424.

[11] Norman J. Pullman, "Clique coverings of graphs IV: algoritinms", SIAM J. Comp. (to appear).

[12] Norman J. Pullman and Dom de Caen, "Clique coverings of graphs III: clique coverings of regular graphs", Congr. inumer. 29 (1980), 795-808.

[13] Norman J. Pul Iman and Dom de Caen, "Clique coverings of graphs - I: clique partitions of regular graphs", Utilitas Math. 19 (1981), $177-205$.

[14] Norman J. Pullman and Alan Donald, "Clique coverings of graphs - II: complements of cliques", Utilitas Math. 19 (1981), 207-213.

[15] H.J. Ryser, "Intersection properties of finite sets", J. Combin. theory Ser. A 14 (1973), 79-92. 
[16] H. Ryser, "Intersection properties of finite sets", Colzoquio Internazionale sulze Teorie Combinatorie (Rome 1973), II, 327-334 (Atti dei Convegni Lincei, No. 17, Accad. Naz. Lincei, Rome, 1976).

[17] Albert Sade, "Enumération des carrés Latins. Applications au $7^{e}$ ordre. Conjecture pour les ordres supérieures" (Published by the author, Marseille, 1948).

[18] S. Vajda, Pattems and configurations in finite spaces (Griffin's Statistical Monographs and Courses, 22. Charles Griffin, London; Hafner, New York; 1967).

[19] Mark B. Wells, "The number of Latin squares of order 8 ", J. Combin. Theory 3 (1967), 98-99.

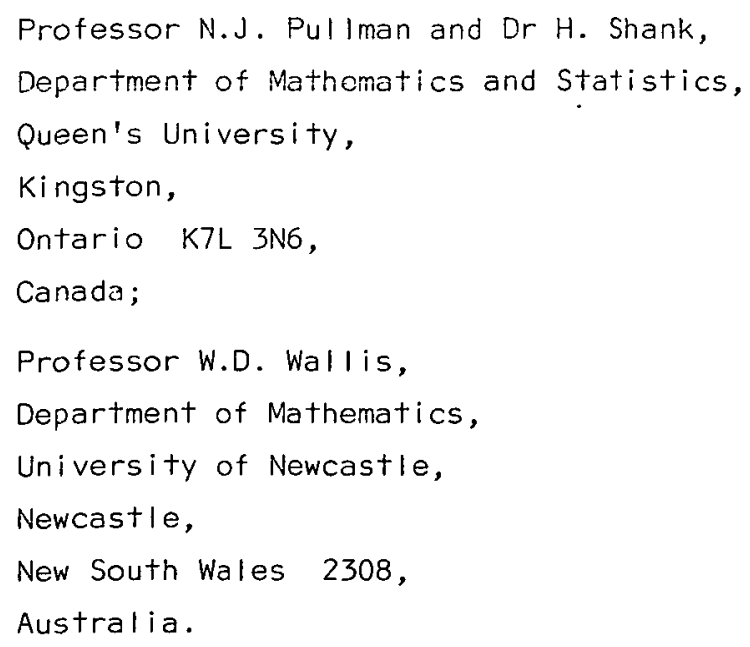

